\title{
Erratum to: A benzimidazopyridoquinoxaline as promising scaffold for G-quadruplex DNA targeting
}

\author{
Asmae Zanzoul ${ }^{1,2,3} \cdot{\text { Youssef } \text { Ramli }^{3,4} \cdot \text { El Mokhtar Essassi }}^{3} \cdot$ Geneviève Pratviel $^{1,2}$
}

Published online: 29 March 2015

(c) Springer Science+Business Media New York 2015

Erratum to: Med Chem Res (2014) 23:4042-4049

DOI 10.1007/s00044-014-0985-1

In the original version of this paper, Dr. Youssef Ramli is affiliated only with Laboratoire de Chimie Organique Hétérocyclique. In addition to this affiliation, he should also be affiliated with the Medicinal Chemistry Laboratory, Faculty of Medicine and Pharmacy, Mohammed V University, Rabat, Morocco.

The online version of the original article can be found under doi:10.1007/s00044-014-0985-1.

Geneviève Pratviel

genevieve.pratviel@1cc-toulouse.fr

1 Laboratoire de Chimie de Coordination, CNRS, UPR 8241, 205 route de Narbonne, BP 44099, 31077 Toulouse Cedex 4, France

2 Université de Toulouse, UPS, INPT, LCC, 31077 Toulouse, France

3 Laboratoire de Chimie Organique Hétérocyclique, pôle de compétences pharmacochimie, Faculté des Sciences,

Université Mohammed V-Agdal, Avenue Ibn Battouta, BP 1014, Rabat, Morocco

4 Medicinal Chemistry Laboratory, Faculty of Medicine and Pharmacy, Mohammed V University, Rabat, Morocco 